\title{
Lymphangioleiomyomatosis: An unusual age of diagnosis with literature review
}

\author{
Youssef Alaoui Lamrani ${ }^{1}$, Badr Alami ${ }^{1}$, Ferdaous Sahnoun ${ }^{2}$, Meriem Boubbou ${ }^{1}$, I mane Kamaoui ${ }^{1}$, \\ Mustapha Maâroufi ${ }^{1}$, Nadia Sqalli Houssaini ${ }^{1}$, Bouchra Amara ${ }^{2}$, Siham Tizniti ${ }^{1}$ \\ 1. Department of Radiology, University Hospital of Fez, Fez, Morocco. 2. Department of Lung Disease, University Hospital \\ of Fez, Fez, Morocco
}

Correspondence: Youssef Alaoui Lamrani. Address: CHU Hassan II, Km 2,200, Route Sidi Hrazem. BP30000, Fez, Morocco. Email: alaouilamraniyoussef@gmail.com

Received: January 6, 2014

Accepted: February 24, 2014

Online Published: February 27, 2014

DOI : $10.5430 /$ ijdi.v1n1p17

URL: http://dx.doi.org/10.5430/ijdi.v1n1p17

\section{Abstract}

Lymphangioleiomyomatosis (LAM) is a rare idiopathic disorder of unclear origin, which almost exclusively occurs to women before menopause. It is characterized by nonneoplastic diffuse proliferation of atypical immature smooth muscle cells around small airways and vessels. We report the case of a 64-year-old woman kept for chronic progressive dyspnea related to a diffuse LAM.

The discussion includes a literature review that describes the physiopathology, clinical features, chest computed tomography aspects, and differential diagnosis regarding this rare disease.

Through this case report, pulmonary LAM should be included in the list of differential diagnoses for cases of pulmonary cystic lesions, even in postmenopausal women.

\section{Keywords}

Lymphangioleiomyomatosis, Postmenopausal diagnosis, Computed tomography, Differential diagnosis

\section{I ntroduction}

Lymphangioleiomyomatosis (LAM), first described in 1937 by Von Stossel, is an uncommon idiopathic disorder of unknown origin affecting women of childbearing age, and patients of both sexes struck by tuberous sclerosis, characterized by a proliferation of smooth muscle cells in the lungs, and vessels ${ }^{[1]}$. The reported prevalence of LAM is approximately one per million, although the true prevalence is likely to be greater ${ }^{[2]}$. LAM is almost exclusively concerned to females, the mean age of onset being 30 years, one third of the cases arise in women less than 20 years of age, and presentation after the menopause is very unusual ${ }^{[3]}$. While LAM is a systemic disease, the main manifestations are pulmonary. In lungs, the atypical progressive proliferation is seen around small airways giving rise to cystic pulmonary changes and progressive respiratory failure ${ }^{[1]}$. 


\section{Case presentation}

We report the case of a 64-year-old woman, referred to our department to explore a chronic and progressive dyspnea appeared 34 years ago, and is actually at stage III according to Sadoul stadification; her past medical history noted menopause around 44 years, and included 10 years of passive smoking. Physical examination showed cyanosis of nail beds, and revealed inspiratory crackles over both lungs. Her oxygen saturation was $87 \%$ in ambient air. A complete blood count revealed polycythemia with hemoglobin concentration of $17.7 \mathrm{~g} / \mathrm{dl}$ (hematocrit of 52.2\%). Initially, posteroanterior chest X-ray radiography was performed, and showed symmetrical, diffuse, reticular interstitial pattern with preservation of lung volume. This radiography was followed by chest CT-scan, which demonstrated the presence of cysts distributed diffusely throughout the lung, well circumscribed, round and oval, of varying sizes and thin-walled. Pulmonary function tests did not reveal obstructive or restrictive pattern. Diagnosis of LAM was made on the basis of clinical and radiologic findings, without resorting to lung biopsy. Abdominal ultrasonography and CT were performed, and ruled out any subclinical renal angiomyolipomas. Continuous home oxygen therapy was indicated and the patient is still in regular follow-up visit.

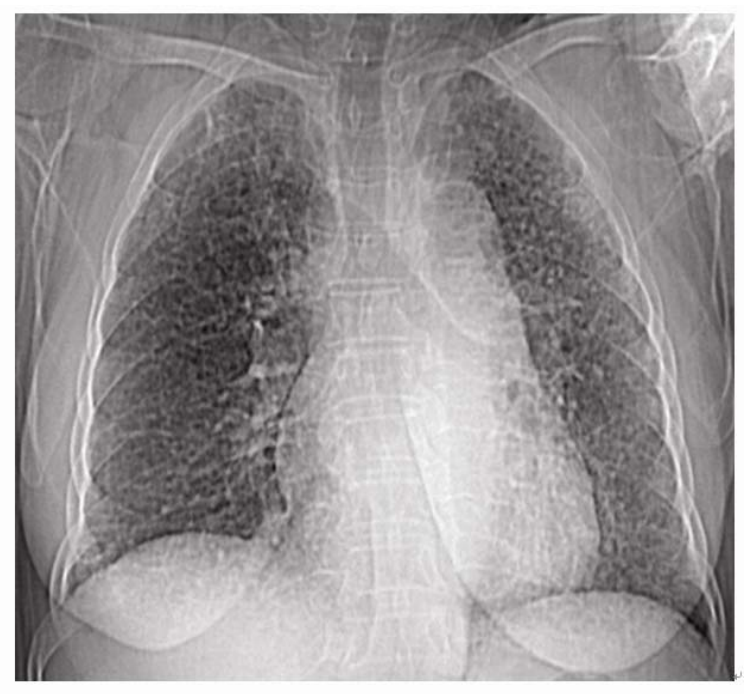

Figure 1. Bilateral symmetric and diffuse reticular interstitial pattern throughout both lung fields with preservation of lung volume.

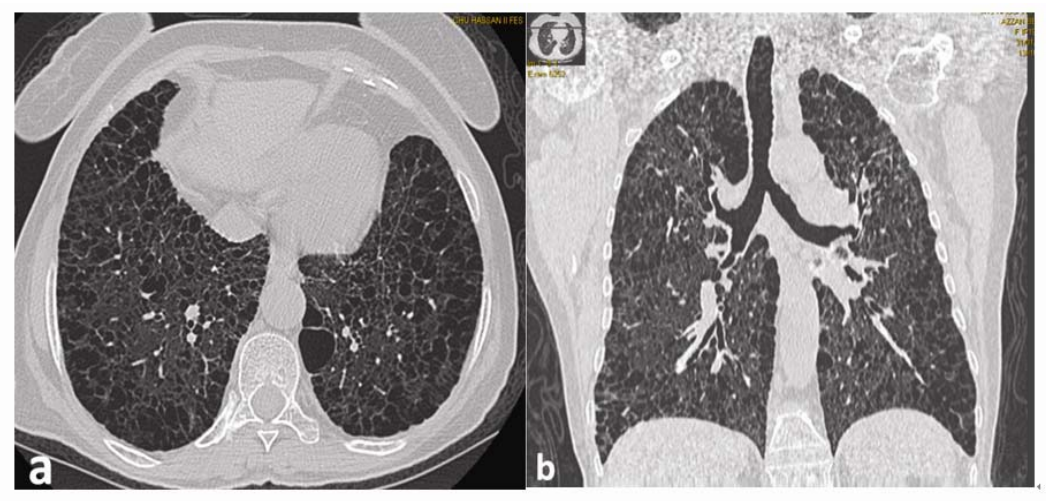

Figure 2. Axial (a) and coronal reformatted image (b) of high-resolution chest CT scan (lung windows) showing near complete replacement of both lungs with thin-walled cysts uniformly distributed giving "lace appearance". 


\section{Discussion}

LAM is a rare disease characterized histologically by a hamartomatous proliferation of smooth muscle principally in the lungs (around small airways, venules, and lymphatic vessels), in mediastinum and abdomen. This proliferation is leading to progressive obstruction of bronchioles, air trapping, and formation of bullae. Obstruction of lymphatics may result in chylothorax and chylous ascites. Obstruction of venules may result in hemosiderosis and hemoptysis ${ }^{[3]}$. LAM can arise sporadically without evidence of other disease in women of childbearing age, or in both sexes in conjunction with tuberous sclerosis complex (TSC) ${ }^{[5]}$. Radiological features consistent with LAM can be found in around 34\% of adult females with TSC $^{[5]}$. The average age at diagnosis is $41-43$ years with first presentation in the late 30s. Presentation after menopause is extremely rare and almost exclusively associated with oestrogen replacement therapy. However, a recent survey suggests that an increasing number of older females are being diagnosed with $\mathrm{LAM}^{[3]}$.

The aetiology of LAM remains obscure; however, the fact that the condition occurs primarily to women who are premenopausal and is exacerbated by high estrogen states suggests a role for hormones in this condition. The link TSC suggests a genetic component. The finding of sporadic LAM in preadolescents, and post menopausal women and even males indicates that estrogens are not the only etiological factor.

LAM follows an insidious course and the rate of progression is variable ranging from a few years to over three decades before culminating in respiratory failure. The disease commonly presents with symptoms of progressive dyspnea, cough, recurrent pneumothorax, or chylothorax (related to obstruction of the thoracic duct or the lymphatics in the pleura). Other manifestations including chest pain, haemoptysis, chyloptysis, and chylopericardium are less common ${ }^{[6]}$. Exacerbations of LAM are described during pregnancy, menstruation, and by exogenous estrogen used for contraception. Extrapulmonary usual documented LAM symptoms may include chylous ascites, hematuria, chyluria, and sudden abdominal bleeding. These abdominal manifestations are related to lymphadenopathy, and lymphangioleiomyomas commonly in the upper retroperitoneum and pelvis; hematuria and abdominal hemorrhage are due to angiomyolipomas that affect predominantly kidneys ${ }^{[6]}$.

Lung function is normal in early disease but patients generally develop progressive airflow obstruction and a decreased lung diffusion capacity. Restrictive defects are usually the result of pleural effusions, surgery or pleurodesis ${ }^{[7]}$.

Chest radiographs often appear normal in more than 95\% of patients in early stages of LAM disease, or may show paradoxical enlarged lung volumes, or may describe a symmetrical, diffuse, reticular interstitial pattern, caused by summation of multiple cyst walls. Computed tomography (CT) scans show numerous thin-walled, well circumscribed, round or oval cysts throughout the lungs, with no predilection for any region of the lung. These cysts vary in size from 2 $\mathrm{mm}$ to $5 \mathrm{~cm}$, as the disease progresses, size and number of cysts increase and have a tendency to conflate, and correlate with disease severity defined by laboratory tests, in particular, decline in DLCO (Carbon Monoxide Diffusing Capacity) and FEV1 (Forced Expiratory Volume in one second). The lung parenchyma in between the cysts is usually inconspicuous, but, in the highly cellular forms of LAM, small nodules, reticular opacification, and ground-glass attenuation can be found ${ }^{[8]}$. Spontaneous pneumothorax is common in LAM, and occurs in about $40 \%$ of patients within the course of the disease ${ }^{[2]}$. Pleural chylous effusions (are indistinguishable from protein-rich effusions of other origin on CT), dilatation of the thoracic duct, and mediastinal adenopathy are further thoracic findings in some patients. In addition, CT shows renal angiomyolipomas in more than $70 \%$ of patients, which have a typical fatty texture, and demonstrates larger fluid-filled cystic masses related to lymphangioleiomyomas in about $16 \%$ of cases. Pancreatic, adrenal, or uterine angiomyolipomas are additional extrathoracic CT findings in some rare cases. These abdominal findings can pre-date respiratory manifestations by many years. For older women, the disease is presented with similar symptoms compared to those in young women, with the exception that the clinical course is longer and more benign after menopause ${ }^{[3]}$.

The most important differential diagnoses for LAM are Langerhans Cell Histiocytoses (LCH), idiopathic pulmonary fibrosis, and panlobular emphysema. In contrast to LAM, in LCH, the costophrenic sulci are usually spared, the cysts are 
smaller, irregular of various shapes, with variable wall thickness, and nodules are predominant in the early stage of disease ${ }^{[2]}$. Idiopathic pulmonary fibrosis shows a volume loss in contrast to LAM, with predominantly basal and subpleural reticulation associated with traction bronchiectasis and basal honeycombing ${ }^{[2]}$. Panlobular emphysema is associated with alpha-1-antiprotease deficiency. The most distinct feature of emphysema is the absence of definable walls in the areas of low attenuation, whereas cysts in LAM are almost invariably present with walls ${ }^{[2]}$. Less common diseases that can mimic LAM and should also be considered include Sjögren syndrome (Older women than LAM), follicular bronchiolitis and lymphocytic interstitial pneumonitis, hypersensitivity pneumonitis, amyloidosis, light chain-deposition disease, bronchopulmonary dysplasia, laryngotracheal papillomatosis, and Birt Hogg Dubé syndrome ${ }^{[2,9]}$. From this wide spectrum of diseases characterized by lung cysts, ranging from isolated chest disorders to rare multisystem diseases, we conclude that LAM's diagnosis has become a complex diagnostic challenge, forcing every clinician to know adequately the natural history of the disease and imaging result that represent the most important key of the diagnosis exercise.

The exact diagnosis of LAM is based on histological study. Generally, lung tissue samples can either be obtained endobronchially, transbronchially, or surgically, but occasionally from lymph nodes or lymphangioleiomyomas. Pathological diagnosis relies on characteristic LAM cell morphology and positive immunoreactivity to smooth muscle actin and HMB-45 antibodies. In a significant proportion of patients, a combination of medical history and high-resolution computed tomography scanning features allows a confident diagnosis to be made, thus obviating the need for biopsy ${ }^{[4]}$.

In the past, LAM treatment strategies were empiric and unproven, based exclusively on the antagonism of estrogen ${ }^{[10]}$. Recent multicentre analysis suggests that LAM is caused by abnormal cell multiplication control. The immunosuppressant rapamycin (macrolide antibiotic), has shown potential to correct this dysfunction. LAM patients who used rapamyxin showed a constant improvement in spirometry after interruption of the treatment. For patients with severe disease, lung transplant should be considered ${ }^{[3]}$.

We conclude that LAM should be considered classically in women of childbearing years with multiple pulmonary cysts, with progressive dyspnea, in women who present with unexplained recurrent pneumothoraces, or chylous effusion. Clinicians should look for LAM in patients with TSC or angiomyolipoma of the kidneys. We recommend radiologists to include pulmonary LAM diagnosis in cases with multiple pulmonary cysts even in postmenopausal women.

\section{Reference}

[1] Marie I, Cailleux N, Henry J, Janvresse A, Muir JF, Lévesque H, Courtois H. Lymphangioleïomyomatose pulmonaire : une déouverte souvent fortuite. A propos d'une observation. Rev Méd Inteme. 1999; 20: 806-9. http://dx.doi.org/10.1016/S0248-8663(00)88689-0

[2] Gurney, Jud W. Et al; Diagnostic Imaging: Chest. First Edition. Amirsys Inc., 2006; Part IV-1-2.

[3] Soler-Ferrer C, Gómez-Lozano A, Clemente-Andrés C, De Cendra-Morera E, Custal-Teixidor M, Colomer-Pairés J. Lymphangioleiomyomatosis in a Post-Menopausal Women. Arch Bronconeumol. 2010 Mar; 46(3): 148-50. http://dx.doi.org/10.1016/S1579-2129(10)70036-7

[4] Johnson SR, Cordier JF, Lazor R, Cottin V, Costabel U, Harari S, Reynaud-Gaubert M, Boehler A, Brauner M, Popper H, Bonetti F, Kingswood C; Review Panel of the ERS LAM Task Force. European Respiratory Society guidelines for the diagnosis and management of lymphangioleiomyomatosis. Eur Respir J. 2010; 35: 14-26. http://dx.doi.org/10.1183/09031936.00076209

[5] Costello LC, Hartman TE, Ryu JH. High frequency of pulmonary lymphangioleiomyomatosis in women with tuberous sclerosis complex. Mayo Clin Proc. 2000; 75: 591-594. http://dx.doi.org/10.4065/75.6.591

[6] Mc Cormack FX Lymphangioleiomyomatosis: a clinical update. Chest. 2008 Feb; 133(2): 507-16. http://dx.doi.org/10.1378/chest.07-0898

[7] Taveira-DaSilva AM, Steagall WK, Rabel A, Hathaway O, Harari S, Cassandro R, Stylianou M, Moss J. Reversible airflow obstruction in lymphangioleiomyomatosis. Chest. 2009 Dec; 136(6): 1596-603. http://dx.doi.org/10.1378/chest.09-0624

[8] D P Tashkin. Lymphangiomyomatosis: CT, chest radiographic, and functional correlations. Radiology. 176: 381-387

[9] Beddy P, Babar J, DevD R Aberle, D M Hansell, K Brownaraj A. A practical approach to cystic lung disease on HRCT. Insights into Imaging. 2011; 2:1-7. http://dx.doi.org/10.1007/s13244-010-0050-7

[10] Kinder BW, Sherman AC, Young LR, Hagaman JT, Oprescu N, Byrnes S, McCormack FX. Predictors for clinical trial participation in the rare lung disease Lymphangioleiomyomatosis. Respir Med. 2010 Apr; 104(4): 578-83. http://dx.doi.org/10.1016/j.rmed.2009.09.023 